\title{
Greater biodiversity in regenerated native tropical dry evergreen forest compared to non-native Acacia regeneration in Southeastern India
}

\section{Christopher Frignoca* and John McCarthy}

Deparment of Atmospheric Science and Chemistry,

Plymouth State University,

Plymouth, New Hampshire, USA

Email: cfrignoca@gmail.com

Email: jcmccarthy177@gmail.com

*Corresponding author

\section{Aviram Rozin}

\author{
Sadhana Forest, \\ Auroville, Tamil Nadu, India \\ Email: aviram@sadhanaforest.org
}

\section{Leonard Reitsma}

Department of Biological Sciences,

Plymouth State University,

Plymouth, New Hampshire, USA

Email: leonr@plymouth.edu

\begin{abstract}
The $20 \mathrm{~km}-50 \mathrm{~km}$-wide belt of tropical dry evergreen forest inland from the southeastern coastline of India has undergone biodiversity loss due to timber harvest and agriculture in the last 200 years. Reforestation restores ecosystem function and increases population sizes and diversity. Sadhana Forest reforested an area of 28 ha and replenished the water table through intensive soil moisture conservation. Results show rapid growth of planted native species and germination of two species of dormant Acacia seeds. Using standardised inventory methods, we documented 75 bird, eight mammal, 12 reptile, five amphibian, 55 invertebrate species, and 22 invertebrate orders. Bird abundance at point count stations, invertebrate sweep net captures and leaf count detections, and Odonate and Lepidopteran visual detections along fixed-paced transects were significantly greater in areas with native plants. Sadhana Forest's reforestation demonstrates the potential to restore ecosystems and replenish water tables, vital components to reversing ecosystem degradation, and corroborates reforestation efforts in other regions of the world.
\end{abstract}

Keywords: reforestation; water conservation; forest regeneration; biodiversity; tropical dry evergreen forest; TDEF; Tamil Nadu; India. 
Reference to this paper should be made as follows: Frignoca, C., McCarthy, J., Rozin, A. and Reitsma, L. (2021) 'Greater biodiversity in regenerated native tropical dry evergreen forest compared to non-native Acacia regeneration in Southeastern India', Interdisciplinary Environmental Review, Vol. 21, No. 1, pp.1-18.

Biographical notes: Christopher Frignoca is a Plymouth State University (PSU) alumnus that received a dual degree in Environmental Biology and Biochemistry. He started his career as a horticultural biologist, conducting research on plant animal co-occurrences in the context of system integrity. He then worked with Canada warblers by determining their breeding success rates and collecting samples for a genoscape project. He has helped lead a research study in Tamil Nadu, India where he performed a bio-inventory on a rehabilitated plot of land and won the PSU 2019 Research Service Award.

John McCarthy studied Environmental Biology for four years at the Plymouth State University. He helped lead a research project in Tamil Nadu, India to promote and study reforestation efforts. He performed independent avian research on foraging differences between flycatcher species and the flocking behaviours of migrant and resident warbler species in Costa Rica. He has been a part of a long-term population study on the Canada Warbler in Canaan, NH. He also contributed to two thesis projects that studied avian community-level diversity changes in response to patch-cutting methods on an American Tree Farm in New Hampshire. He has also volunteered on a raptor banding project in Cape May, NJ.

Aviram Rozin is the Founder and International Director of Sadhana Forest, a volunteer-based organisation focused on creating long-term food security through environmental restoration. He is a member of the Global Restoration Council and a board member of the Foundation for World Education. Globally, 133 million malnourished people are living in arid areas, have private land, but are not using it to grow food due to lack of water and agricultural knowledge. Sadhana Forest trains local people in India, Haiti and Kenya in the use of water-saving irrigation techniques and provides them with free seedlings to plant drought-resistant, indigenous, food-bearing trees around their homes. These food forests are well protected from cutting and animal grazing by their owners. Sadhana Forest won third prize at The Humanitarian Water and Food Awards. Every year the organisation's India, Haiti and Kenya centres host and train a total of over 1,500 volunteers, students, and interns.

Leonard Reitsma earned his BS in Biology at the William Paterson University in Wayne, NJ in 1985 and his PhD from the Dartmouth College, Hanover, NH in 1990. He has been a Professor at the Plymouth State University since 1992 teaching ornithology, vertebrate zoology, tropical biology, conservation, ecology and current environmental issues. He has researched avian population ecology since the mid-1980s and is currently working on a long-term population study of Canada warblers in Canaan, $\mathrm{NH}$ as well as a community-level project monitoring avian species diversity changes in response to small patch cuts on the American Tree Farm he owns with his family. 


\section{Introduction}

Human population growth and agricultural expansion has been identified as the driving force in deforestation on the local, regional, and global scale (Acheampong et al., 2019; Binsangou et al., 2018; Carr, 2009; Geist and Lambin, 2002; Oyetunji et al., 2020). Deforestation in India has been a problem for over 200 years and has led to species extinction and extirpation mostly due to loss of habitat. Between 1823 and 1850 up to $50 \%$ of India's forests disappeared due to the East India Company, Royal Navy, and Company Marine clear-cutting for cash crops (Grove, 2002; Babu et al., 2019; Pyles et al., 2018). In more recent history, from 1930-2013, $28 \%$ of additional forest has been cut down due to rising human population and the consequent demand for land mostly to produce crops (Pyles et al., 2018; Reddy et al., 2016, 2018).

Tropical dry evergreen forests (TDEFs) have been heavily impacted by deforestation and the species in these systems are in major threat of extinction (Muthumperumal and Parthasarathy, 2016; Babu et al., 2019; Pyles et al., 2018). TDEF are two-layered evergreen forests that experience six dry months a year (Muthumperumal and Parthasarathy, 2016). They are one of the 16 minor and 6 major forest types in India and are located within a narrow band along the southeast corridor of India. This forest type is being threatened by urbanisation, agriculture, and climate change resulting in a species extinction rate of around $0.8 \%-2 \%$ per year (Everard, 2018; Nithaniyal et al., 2017). TDEF only grow on a belt of vegetation between 20 and $50 \mathrm{~km}$ wide inland from the southeast coastline of India, and this narrow distribution coupled with the coastal proximity makes them particularly vulnerable to human impact (Muthumperumal and Parthasarathy, 2016; Pyles et al., 2018; Reddy et al., 2018).

Auroville has responded proactively to this TDEF deforestation. When the community of Auroville started in 1968, the land was a dry plateau of dirt that had very few trees. The trees were cut for timber export and agriculture 200 years ago, and with long dry seasons, the land experienced damaging periods of monsoon and erosion (Everard, 2018; Grove, 2002). Organisations in Auroville are involved with water conservation, reforestation, and increasing biodiversity.

Sadhana Forest is an organisation within Auroville that was founded in 2003 with the primary focus of reforestation and water conservation. Water runoff has been reduced and soil moisture increased by digging $30 \mathrm{~km}$ of on-contour swales and bunds. When soil moisture increased, trees started naturally regenerating. Two species of non-native Acacia appeared: earpod wattle (Acacia holosericeal) and strap wattle (Acacia auriculiformis). These seeds were spread on the land in the early 1980s by a forester from Auroville who hoped to prevent erosion and reforest the land with trees from dry habitats, but the seeds entered dormancy due to low soil moisture. Sadhana Forest has planted 170 different native TDEF plant species in hopes of restoring natural habitat and increasing biodiversity.

Non-native plants can pose a threat to native biodiversity throughout trophic chains (Johnson, 2007; Babu et al., 2019; Tallamy, 2004), and biodiversity is commonly documented to be lower for bird and invertebrate species in less native habitats (Holmes and Schultz, 1988; Johnson, 2007; Narango et al., 2017; Tallamy, 2004). However, nonnative plants can promote increases in other food items, such as non-native arthropods, and keep the biomass of prey equivalent to a system dominated by native plant species (Narango et al., 2017). Non-native Acacia unexpectedly regenerated in certain areas of Sadhana Forest when the water table increased, and the effect on other trophic levels of 
these non-natives is unknown. Do these novel ecosystems with a mix of native and nonnative plants represent a necessary compromise given the multi-century history of this area? (Hobbs et al., 2014; Kueffer and Kaiser-Bunberry, 2014) There is precedent for novel ecosystems with mixes of native and non-native species having greater diversity, nutrient cycling and total plant biomass (Mascaro et al., 2012). Areas reforested in predominantly Acacia spp. may still provide habitat for native fauna returning to previously deforested areas, but how this compares to recruitment and immigration of native fauna to areas intentionally planted with native species needs further examination. We hypothesised that 15 years of forest regeneration will result in high diversity of fauna on the reforested parts of the Sadhana Forest property, and locations within the property with a higher proportion of native plants will have higher faunal diversity than those dominated by non-native Acacia species, despite outcomes from restoration leading to novel ecosystems.

\section{Methods}

\subsection{Study site}

This study was conducted within Sadhana Forest, Auroville, Tamil Nadu, India $\left(11.9807^{\circ} \mathrm{N}, 79.7766^{\circ} \mathrm{E}\right)$. The area consists of 28.3 hectares of TDEF containing over 170 different species of six plant types: trees, shrubs, lianas, epiphytes, herbs, and tuberous species. The site was delineated into 17 zones A-J (Figure 1) to organise the rehabilitation of the land. Sadhana Forest has risen the water table, causing natural regrowth throughout the property, and some zones have received more long-term reforestation efforts [residential (Res), B, E, G, H, J], than others (A, C, I, K, M, N, O). Zones with long-term efforts consist of greater amounts of native plant species when compared to other zones, which have lower stem densities and more non-native Acacia trees. Zones D and $\mathrm{F}$ have had no reforestation efforts. We established 38 sampling locations throughout the property excluding two pieces of land, SFProperty, due to its recent acquisition and unsurveyed property line on the original map provided by Sadhana Forest, and Children's Land (ChLand), because of the consistent recreational activities occurring there (Figure 1). Sampling locations were placed at 110-150 m intervals to minimise duplicate detection while stratifying the sampling across the entire forest.

\subsection{Cumulative count}

To compile a comprehensive and cumulative list of all species identified, we recorded all species within all zones of the Sadhana Forest property from 7 July-16 August 2018 and included those seen, heard, or photographed by any volunteers at Sadhana Forest.

\subsection{Point count}

We recorded all individuals of all bird species at each sampling location by performing point counts. We visited each grid point beginning around 5:30 and stopped at around 9:00. In each zone we recorded all species seen or heard within a 50m radius at each grid point for two consecutive periods of $5 \mathrm{~min} 30 \mathrm{~s}$, mapping each detection by estimated distance and direction from the sampling location. We did the same for individuals 
outside the $50 \mathrm{~m}$ radius or individuals flying over during the duration of the point count. We conducted three point counts at each sampling location with a minimum of one week between counts.

Figure 1 Map of Sadhana Forest that includes the property boundaries and zone differentiation outlined in red (see online version for colours)
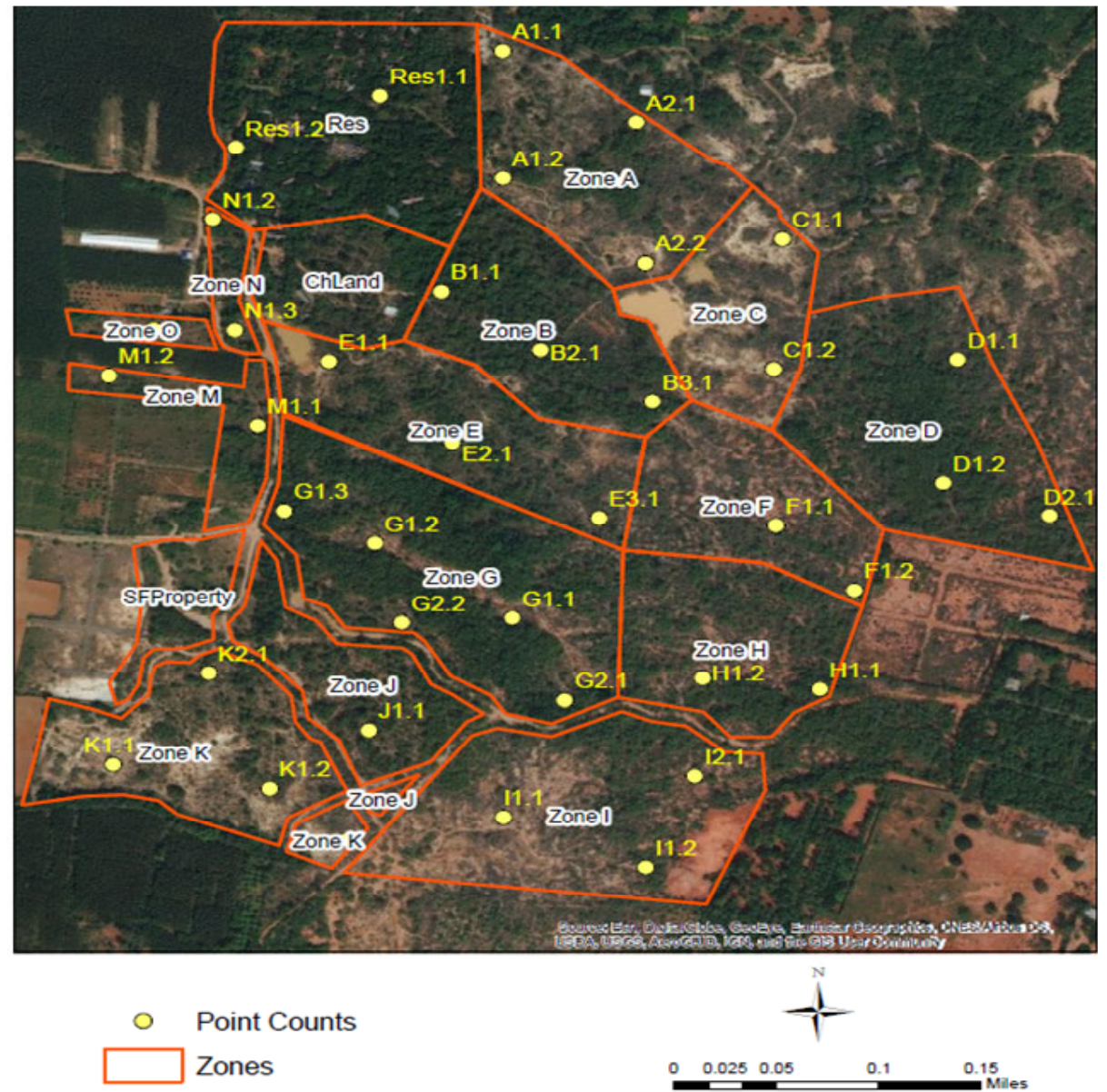

Notes: Stratified sampling locations, marked by yellow circles, show areas where data were collected for point counts, sweep netting, leaf counts, and vegetation measurements. Other data were collected while walking throughout the property. Sadhana Forest Property (SFProperty) and Children's Land (ChLand) were not used for any sampling methods except for cumulative counts.

\subsection{Sweep netting}

We performed sweep netting within a $50 \mathrm{~m}$ radius at each grid point. We visited each point two times, once in early afternoon with lower perceived predator activity, and once in early morning with presumed higher predator activity. We performed three $10 \mathrm{~m}$ sweeps in the available vegetation, prioritising tall grasses. We swept the net from side to side in a linear path each time in a different direction to prevent overlap. After each $10 \mathrm{~m}$ 
sweep, we emptied the invertebrates into a plastic container in order to identify them. We classified the insects to order, unless a more detailed classification (ex., family, genus, and species) level was known. We also recorded the common name for the type of invertebrate within the order classified (ex., hymenoptera-ant or hymenoptera-wasp); we then released all animals collected as this was completely non-destructive sampling throughout.

\subsection{Leaf counts}

We counted invertebrates on leaf surfaces of the 50 native trees closest to each sampling location. We inspected leaves for invertebrates only on trees taller than $1 \mathrm{~m}$. We stratified each sampled tree into three zones, $\leq 1 \mathrm{~m}, 1-2 \mathrm{~m}$, and $\geq 2 \mathrm{~m}$. We inspected 50 leaves (if available) in each stratum of each tree, for a maximum of 150 leaves per tree. We also counted up to a maximum of 30 of the closest non-native trees, if possible: 15 strap wattle and 15 earpod wattle at each sampling location following the same criteria for counting invertebrates on leaves of the native trees. We recorded insects to order, or a more detailed classification if possible, as with the sweep netting.

\subsection{Lepidoptera and Odonata counts}

To inventory the diversity and abundance of Lepidoptera (butterflies and moths) and Odonata (dragonflies and damselflies), we walked the study site at a fixed pace beginning at 9:00 visiting each sampling zone in the following order: Res, A, C, D, F, H, G, J, G, E, $\mathrm{N}, \mathrm{O}, \mathrm{M}, \mathrm{G}, \mathrm{K}, \mathrm{I}, \mathrm{G}, \mathrm{E}$, and $\mathrm{B}$, while following the easiest path to the next sampling grid location. The reverse order was walked the following day. We walked the study plots in both directions to minimise the influence of environmental factors like sun availability and temperature from influencing detections.

\subsection{Vegetation analysis}

Vegetation was measured within a $25 \mathrm{~m}$ radius around each grid point. We estimated the percent cover of shrub and understory canopy layers, canopy cover with a spherical crown densitometer, and average canopy height at each sampling location. We estimated the percent representation of native species compared to non-native species (earpod and wattle Acacia trees). We recorded the percentage of ground cover within a $5 \mathrm{~m}$ radius around each grid point in the following categories: leaf litter, rock (sand and gravel), herbaceous, and woody debris. All percent cover estimates added to $100 \%$.

\subsection{GIS mapping}

We used ArcGIS (ESRI 2011, ArcGIS Desktop: Release 10, Redlands, CA: Environmental Systems Research Institute) to create maps representing Sadhana Forest (Figure 1). We used georeferencing and coordinate data from a $\mathrm{kml}$ file in order to plot the property boundaries, zone differentiations, and sampling locations. We also created a map to interpolate bird hot spots on the property. We used the spatial analyst interpolation tool in order to represent the cumulative excel point count data at each sampling location in comparison to the surrounding locations. 


\subsection{Statistical analysis}

All analyses were performed in RStudio [R stats version 3.5.2 (R Core Team, 2018)]. We used R version 3.5.2 (20 December 2018) to perform Pearson's product-moment correlations to compare the percentage of native plants to: bird detects and bird diversity from point count data, invertebrate detects from sweep netting, and flying invertebrate detects from fixed-paced survey counts in order to obtain linear regression statistics. We performed a Welch two sample t-test to compare invertebrates found in leaf counts of native plants to those found in Acacia trees.

\section{Results}

We recorded a total of 75 bird species, 55 invertebrate species, 23 invertebrate orders, 8 mammal species, 12 reptile species, and 5 amphibian species (Table 1). Some additional detected species were unable to be classified, such as, 2-3 more bat species and several invertebrate species. Bird detections were significantly more abundant at point counts with more native vegetation ( $\mathrm{df}=36, \mathrm{R}^{2}=0.526, \mathrm{~F}=3.714, \mathrm{p}<0.001$, Figure 2). Bird diversity was not, however, significantly correlated with the proportion of native vegetation $\left(\mathrm{df}=36, \mathrm{R}^{2}=-0.0998, \mathrm{~F}=-0.602, \mathrm{p}=0.551\right.$, Figure 3$)$. Invertebrate sweep netting yielded greater captures in areas with more native plants $\left(\mathrm{df}=36, \mathrm{R}^{2}=0.351\right.$, $\mathrm{F}=2.25, \mathrm{p}=0.031$, Figure 4) and Lepidopteran and Odonate detections during fixed-pace surveys were also greater where there were more native plants $(\mathrm{df}=34$, $\mathrm{R}^{2}=0.430, \mathrm{~F}=2.778, \mathrm{p}=0.0089$, Figure 5). As well, significantly more invertebrates were detected through leaf counts on native trees $(\mathrm{df}=14.285, \mathrm{t}=-6.3703, \mathrm{p}<0.00005$, Figure 6).

Figure 2 The total number of birds detected at each point count location was significantly greater at point count stations with greater native tree cover

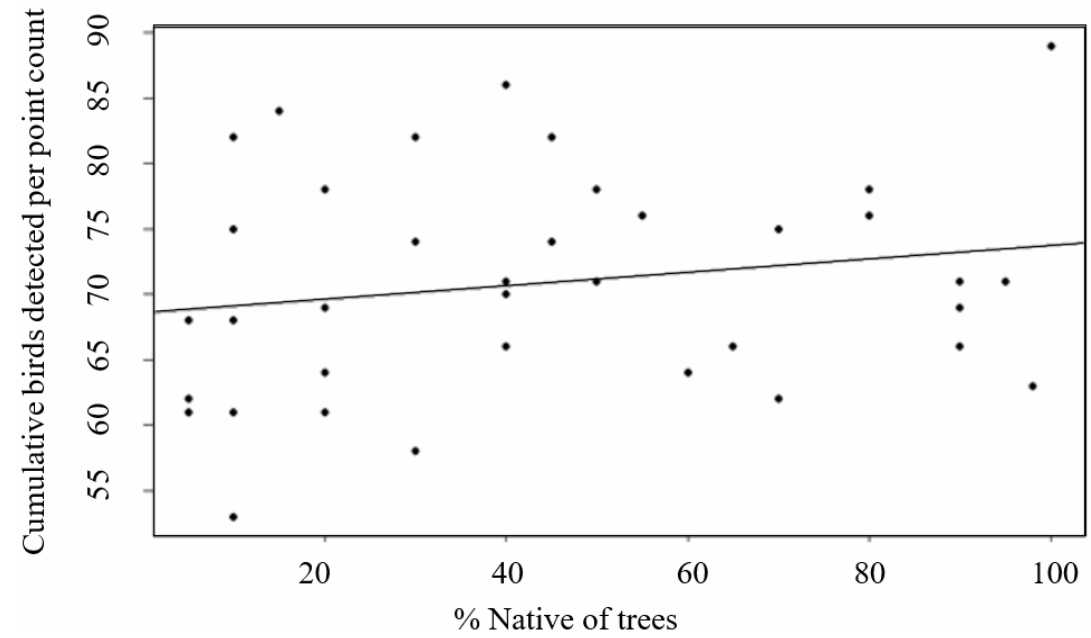

Notes: $\mathrm{df}=36, \mathrm{R}^{2}=0.526, \mathrm{~F}=3.714, \mathrm{p}<0.001$. 
The interpolated bird hotspot map generated through ArcGIS shows highest bird diversity in areas with more native vegetation in relation to the point count data (Figure 7).

Figure 3 The bird diversity detected at each point count did not significantly differ between point counts with more vs. less native tree cover

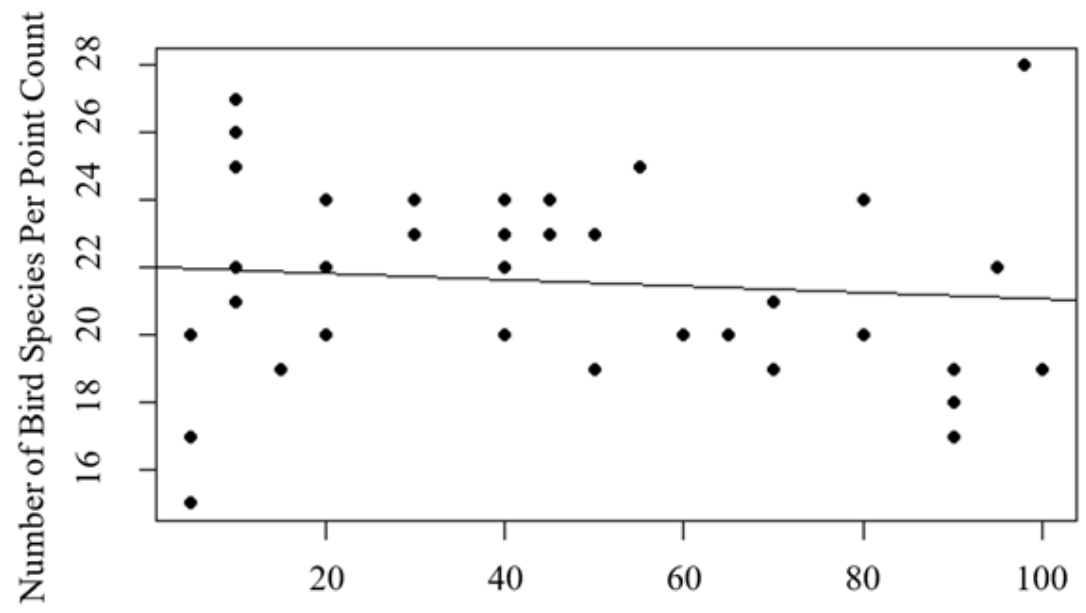

\section{$\%$ Native of Trees}

Notes: $\mathrm{df}=36, \mathrm{R}^{2}=-0.0998, \mathrm{~F}=-0.602, \mathrm{p}=0.551$.

Figure 4 The number of invertebrates captured in sweep net sampling was significantly greater in areas with more native tree cover

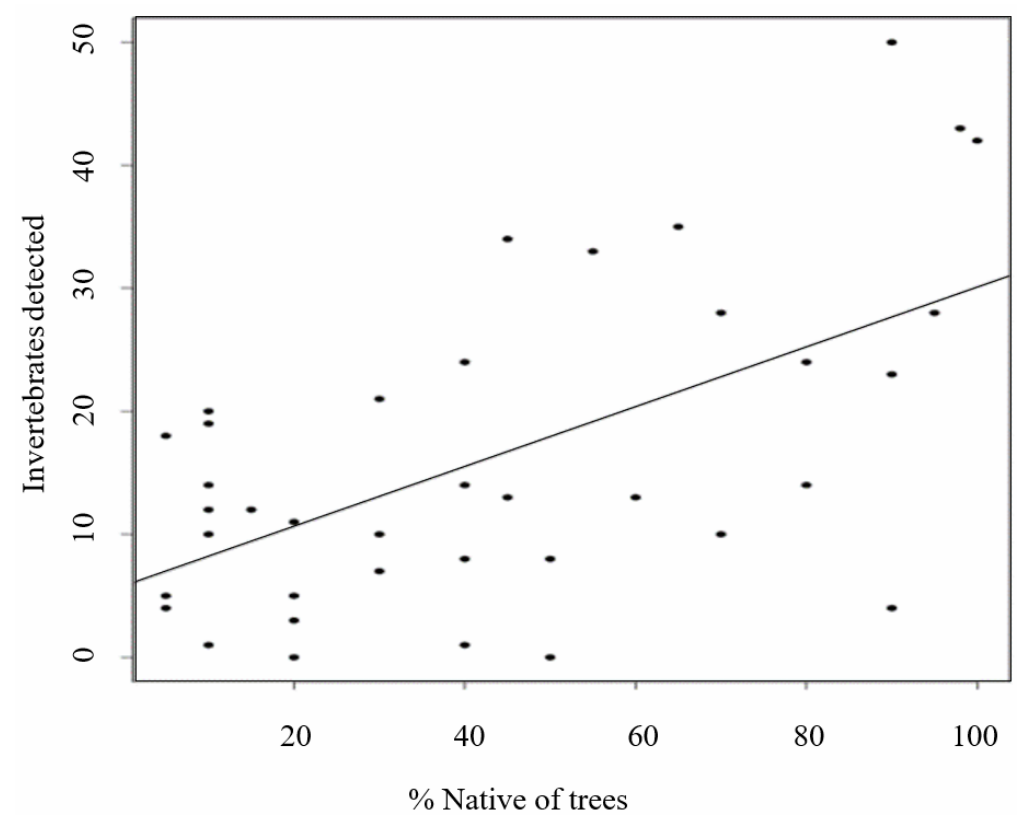

Notes: $\mathrm{df}=36, \mathrm{R}^{2}=0.351, \mathrm{~F}=2.25, \mathrm{p}=0.031$. 
Figure 5 The number of Odonates and Lepidopterans detected during fixed-pace surveys was significantly greater in areas with greater native tree cover

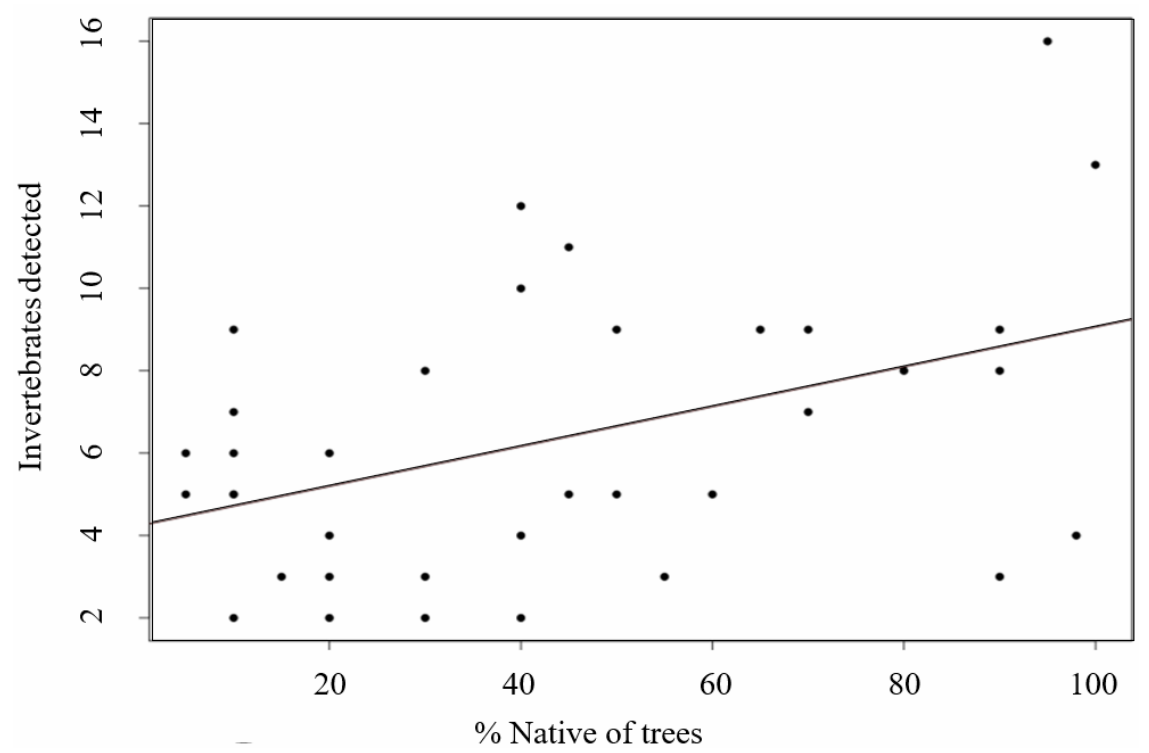

Notes: $\mathrm{df}=34, \mathrm{R}^{2}=0.430, \mathrm{~F}=2.778, \mathrm{p}=0.0089$.

Figure 6 The number of arthropods detected during leaf counts was significantly greater on native trees than Acacia trees

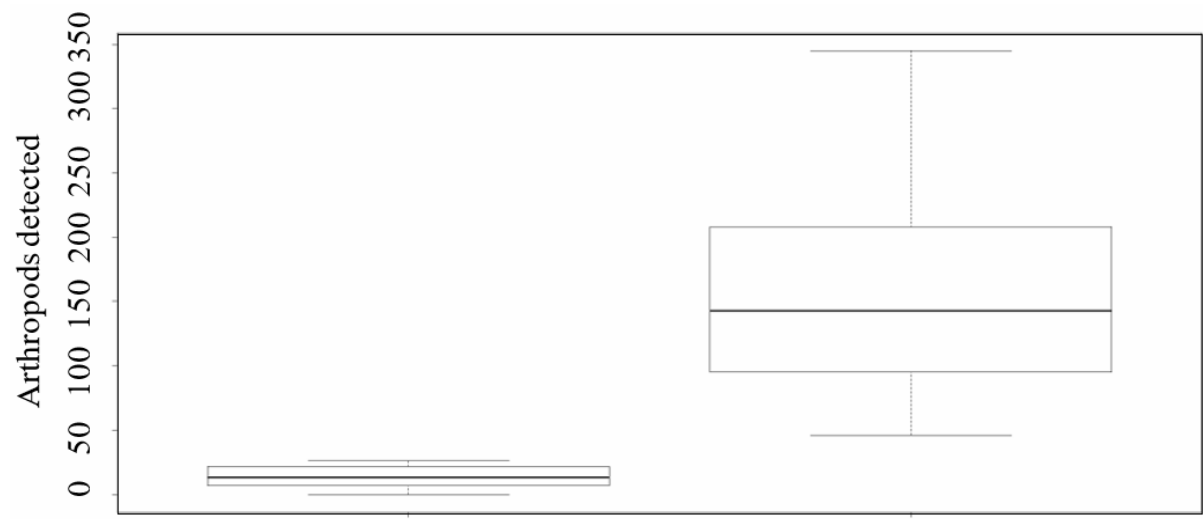

Acacia species

Native species

Cover type

Notes: $\mathrm{df}=14.285, \mathrm{t}=-6.3703, \mathrm{p}<0.0001$. 
Table 1 Comprehensive list of taxa from a bioinventory of Sadhana Forest (7 July-16 August 2018)

\begin{tabular}{|c|c|c|c|}
\hline Sub-phylum & Common name & Species & Order \\
\hline \multirow[t]{38}{*}{ Invertebrates } & Wolf spider & Lycosidae spp. & Araneae \\
\hline & Green lynx spider & Peucitia viridans & Araneae \\
\hline & Huntsman spider & Sparassidae spp. & Araneae \\
\hline & Signature spider & Argiope anasuja & Araneae \\
\hline & Adanson's jumping spider & Hasarius adansoni & Araneae \\
\hline & Indian domino cockroach & Therea petiveriana & Blattodea \\
\hline & Blister beetle & Mylabris pustulata & Coleoptera \\
\hline & Six-spot ground beetle & Anthia sexguttata & Coleoptera \\
\hline & Indian freshwater crab & Travancoriana schirnerae & Crustacean \\
\hline & Yellow-spotted millipede & Harpaphe haydeniana & Diplopoda \\
\hline & Hover fly & Syrphidae sspp. & Diptera \\
\hline & Robber fly & Asilidae spp. & Diptera \\
\hline & Giant African snail & Achatina fulica & Gastropoda \\
\hline & St. Andrew's cotton stainer & Dysdercus andreae & Hemiptera \\
\hline & Mud dauber & Sceliphron caementarium & Hymenoptera \\
\hline & Wasp spp. & Trogaspidia ashmead & Hymenoptera \\
\hline & Weaver ant & Oecophylla spp. & Hymenoptera \\
\hline & Carpenter bee & Xylocopa violacea & Hymenoptera \\
\hline & Greater banded hornet & Vespa tropica & Hymenoptera \\
\hline & Blue banded bee & Amegilla spp. & Hymenoptera \\
\hline & Crimson tip & Colotis danae & Lepidoptera \\
\hline & Common mormon & Papilio polytes & Lepidoptera \\
\hline & Plain orange tip & Colotis aurora & Lepidoptera \\
\hline & Plain tiger & Danaus chryssippus & Lepidoptera \\
\hline & Common castor & Ariadne merione & Lepidoptera \\
\hline & Dakhan common gull & Cepora nerissa phyrne & Lepidoptera \\
\hline & Great eggfly & Hypolimnas bolina & Lepidoptera \\
\hline & Blue mormon & Papilio polymnestor & Lepidoptera \\
\hline & Common blue tail & Ischnura senegalensis & Lepidoptera \\
\hline & Common emigrant & Catopsilia pomona & Lepidoptera \\
\hline & Common leopard & Phalanta phalantha & Lepidoptera \\
\hline & Tawny coster & Acraea terpiscore & Lepidoptera \\
\hline & Common grass yellow & Eurema hecabe & Lepidoptera \\
\hline & Lime butterfly & Papilio demoleus & Lepidoptera \\
\hline & Indian sunbeam & Curetis thetis & Lepidoptera \\
\hline & Crimson rose & Pachliopta hector & Lepidoptera \\
\hline & Hummingbird hawk-moth & Macroglossum stellatarum & Lepidoptera \\
\hline & Blue tiger & Tirumala limniance & Lepidoptera \\
\hline
\end{tabular}


Table 1 Comprehensive list of taxa from a bioinventory of Sadhana Forest (7 July-16 August 2018) (continued)

\begin{tabular}{|c|c|c|c|}
\hline Sub-phylum & Common name & Species & Order \\
\hline \multirow[t]{18}{*}{ Invertebrates } & Lemon pansy butterfly & Junonia lemonias & Lepidoptera \\
\hline & Straight swift butterfly & Parnara spp. & Lepidoptera \\
\hline & Common banded peacock & Papilio crinois & Lepidoptera \\
\hline & Common crow butterfly & Euploea core & Lepidoptera \\
\hline & Yellow pansy & Junonia hienta & Lepidoptera \\
\hline & Plain cupid butterfly & Chilades pandava & Lepidoptera \\
\hline & Blue pansy butterfly & Junonia orythia & Lepidoptera \\
\hline & Death's head hawkmoth & Vitex negundo & Lepidoptera \\
\hline & Praying mantis spp. & Amorphposcelis annulicornis & Mantodea \\
\hline & Indian stick mantis & Aethalochroa insignis & Mantodea \\
\hline & Three-striped blue dart & Pseudagrion decorum & Odonata \\
\hline & Indian skimmer dragonfly & Diplacodes trivialis & Odonata \\
\hline & Megarian banded centipede & Scolopendra cingulata & Scolopendra \\
\hline & Tailless whip scorpion & Amblypygi tailless & Scorpiones \\
\hline & Whip scorpion & Theylyphonida spp. & Scorpiones \\
\hline & Indian black scorpion & Heterometrus spp. & Scorpiones \\
\hline & Indian red scorpion & Hottentotta tamulus & Scorpiones \\
\hline & & Total species: 55 & Total orders: 22 \\
\hline \multirow[t]{9}{*}{ Mammals } & Indian boar & Sus scrofa & \\
\hline & Indian field mouse & Mus booduga & \\
\hline & Indian flying fox & Pteroupus giganteus & \\
\hline & Indian grey mongoose & Herpestes edwardsii & \\
\hline & Indian hare & Lepus nigricollis & \\
\hline & Indian palm squirrel & Funambulus palmarum & \\
\hline & Indian porcupine & Hystrix indica & \\
\hline & Small Indian civet & Viverricula indica & \\
\hline & & Total species: 8 & \\
\hline \multirow[t]{10}{*}{ Birds } & Ashy crowned sparrow lark & Eremopterix griseus & \\
\hline & Asian koel & Eudynamys scolopaceus & \\
\hline & Asian openbill & Anastomus oscitans & \\
\hline & Asian palm swift & Cypsiurus balasienses & \\
\hline & Asian paradise flycatcher & Terpsiphone paradisi & \\
\hline & Baya weaver & Ploceus philippinus & \\
\hline & Black drongo & Dicrurus macrocerus & \\
\hline & Black-rumped flameback & Dinopium benghalense & \\
\hline & Black-crowned night heron & Nycticorax nycticorax & \\
\hline & Blue-faced malkoha & Phaenicophaeus viridirostris & \\
\hline
\end{tabular}


Table 1 Comprehensive list of taxa from a bioinventory of Sadhana Forest (7 July-16 August 2018) (continued)

\begin{tabular}{|c|c|c|c|}
\hline Sub-phylum & Common name & Species & Order \\
\hline \multirow[t]{38}{*}{ Birds } & Brown-headed barbet & Megalaima zeylanica & \\
\hline & Caspian tern & Hydroprogne caspia & \\
\hline & Common babbler & Turdoides caudata & \\
\hline & Common hoopoe & Upapa epops & \\
\hline & Common iora & Aegithina tiphia & \\
\hline & Common kingfisher & Alcedo atthi & \\
\hline & Common myna & Acridotheres tristis & \\
\hline & Common tailorbird & Orthotomus sutorius & \\
\hline & Common wood shrike & Tephrodornis pondicerianus & \\
\hline & Coppersmith barbet & Megalaima haemacephala & \\
\hline & Crested honey buzzard & Pernis ptilorhynchus & \\
\hline & Drongo cuckoo & Surniculus lugubris & \\
\hline & Eurasian collared dove & Streptopelia decaocto & \\
\hline & Greater coucal & Centropus sinensis & \\
\hline & Greater flamingo & Phoenicopterus roseus & \\
\hline & Green bee eater & Merops orientalis & \\
\hline & Grey francolin & Francolinus pondicerianus & \\
\hline & Grey-bellied cuckoo & Cacomantis passerinus & \\
\hline & House crow & Corvus splendens & \\
\hline & Indian cormorant & Phalacrocorax fuscicollis & \\
\hline & Indian golden oriole & Oriolus kundoo & \\
\hline & Indian grey hornbill & Ocyceros birostris & \\
\hline & Indian night jar & Caprimulgus asiaticus & \\
\hline & Indian peafowl & Pavo cristatus & \\
\hline & Indian pond heron & Ardeola grayii & \\
\hline & Indian robin & Saxicoloides fulicatus & \\
\hline & Indian roller & Coracias benghalensis & \\
\hline & Indian silverbill & Eudoice malabarica & \\
\hline & Jacobin cuckoo & Clamator jacobinus & \\
\hline & Jerdon's bushlark & Mirafa affinis & \\
\hline & Jerdon's leafbird & Chloropsis hyperythra & \\
\hline & Jungle babbler & Turdoides striata & \\
\hline & Jungle bush quail & Perdicula asiatica & \\
\hline & Jungle crow & Corvus macrorhynchos & \\
\hline & Jungle prinia & Prinia sylvatica & \\
\hline & Laughing dove & Spilopelia senegalensis & \\
\hline & Loten's sunbird & Cinnyris lotenius & \\
\hline & Oriental magpie robin & Copsychus saularis & \\
\hline
\end{tabular}


Table 1 Comprehensive list of taxa from a bioinventory of Sadhana Forest (7 July-16 August 2018) (continued)

\begin{tabular}{|c|c|c|c|}
\hline Sub-phylum & Common name & Species & Order \\
\hline \multirow[t]{28}{*}{ Birds } & Oriental skylark & Alauda gulgula & \\
\hline & Painted francolin & Francolinus pictus & \\
\hline & Painted stork & Mycteria leucocephala & \\
\hline & Pale-billed flowerpecker & Dicaeum erythrorhynchos & \\
\hline & Pied bush chat & Saxicola caprata & \\
\hline & Plain prinia & Prinia inornata & \\
\hline & Purple sunbird & Cinnyris asiaticus & \\
\hline & Purple-rumped sunbird & Leptocoma zeylonica & \\
\hline & Red jungle fowl & Gallus gallus & \\
\hline & Red-vented bulbul & Pycnontus cafer & \\
\hline & Red-wattled lapwing & Vanellus indicus & \\
\hline & Rose-ringed parakeet & Psittacula krameri & \\
\hline & Rufous tailed lark & Ammomanes phoenicura & \\
\hline & Rufous treepie & Dendrocitta vaganbunda & \\
\hline & Shikra & Accipiter badius & \\
\hline & Sirkeer malkoha & Phaenicophaeus leschenaultii & \\
\hline & Small minivet & Pericrocotus cinnamomeus & \\
\hline & Spotted dove & Spilopelia chinensis & \\
\hline & Spotted owlet & Athene brama & \\
\hline & Tawny-bellied babbler & Dumetia hyperythra & \\
\hline & Thick-billed flowerpecker & Dicaeum agile & \\
\hline & White throated kingfisher & Halcyon smyrensis & \\
\hline & White-bellied drongo & Dicrurus caerulescens & \\
\hline & White-browed bulbul & Pycnonotus luteolus & \\
\hline & White-browed wagtail & Motacilla maderaspatensis & \\
\hline & White-rumped munia & Lonchura striata & \\
\hline & Yellow-billed babbler & Turdoides affinis & \\
\hline & & Total species: 75 & \\
\hline \multirow[t]{9}{*}{ Reptiles } & Bengal monitor & Varanus bengalensis & \\
\hline & Common house gecko & Hemidactylus frenatus & \\
\hline & Common krait & Bungarus caeruleus & \\
\hline & Common wolf snake & Lycodon capucinus & \\
\hline & $\begin{array}{l}\text { Dumeril's black headed } \\
\text { snake }\end{array}$ & Sibynophis subpunctatus & \\
\hline & Fan-throated lizard & Sitana ponticeriana & \\
\hline & Indian chameleon & Chamaeleo zeylanicus & \\
\hline & Indian rat snake & Ptyas mucosa & \\
\hline & Indian skink & Eutropis multifasciata & \\
\hline
\end{tabular}


Table 1 Comprehensive list of taxa from a bioinventory of Sadhana Forest (7 July-16 August 2018) (continued)

\begin{tabular}{lccc}
\hline Sub-phylum & Common name & Species & Order \\
\hline Reptiles & Olive keelback & Artretium schistosum \\
& Oriental garden lizard & Calotes versicolor \\
& Russell's pit viper & Daboia russelii \\
& & Total species: 12 \\
Amphibians & Common Indian tree frog & Polypedates leucomystax \\
& Indian green frog & Euphlyctis hexadactylus \\
& Indian skipper frog & Euphlyctis cyanophylctis \\
& Indian toad & Duttaphrynus melanostictus \\
Unknown & Sri Lankan painted frog & Kaloula taprobanica \\
Taxa & Bat species & Total species: 5 \\
& Several invertebrate & \\
\hline
\end{tabular}

\section{Discussion}

Our standardised measures of faunal diversity and abundance coupled with the cumulative list of taxa detected over the six weeks of our bioinventory indicate Sadhana Forest's reforestation has attracted a diverse community of vertebrate and invertebrate species. Our results also support our hypothesis showing that areas with higher amounts of native vegetation have higher faunal diversity and abundance. Although bird diversity regressed with the proportion of native plants at each point count did not show a significant correlation, bird diversity hotspots were associated with areas with more native vegetation - see below. Several studies have documented lower biodiversity in areas with comparatively fewer native plant species (Holmes and Schultz, 1988; Johnson, 2007; Narango et al., 2017; Tallamy, 2004). Cristescu et al. (2012) also documented lower faunal diversity in areas disturbed by mining operations compared to nearby undisturbed areas, but mining presents a particular restoration challenge, albeit an important challenge (Palmer et al., 2010; Prach et al., 2011). However, this is not a universal pattern. Mascaro et al. (2012) documented higher diversity in Hawaiian forests with a mix of native and non-native species than in those with only native species. From a forest restoration perspective, careful consideration must be given to efforts to regenerate native forests or accept a new mix of species in what are currently referred to as novel ecosystems (Murcia et al., 2014). This is particularly relevant to the TDEF at Sadhana because removing Acacia would take significant effort. Clearly, the water retention success at Sadhana is largely responsible for both the success in reforesting through active native plantings and germination of dormant Acacia seeds (Prach and Hobbs, 2008).

We did not find a significant relationship between the proportion of native plant species at each point count location and bird diversity, although there was a positive influence on bird abundance. As stated, this analysis was based upon our estimates of the 
proportion of native plants at each point count station while in a subsequent effort to render bird diversity throughout the entire property using ArcGIS, we found that the diversity hotspots did coincide with those areas with greater native plant cover. Due to the high mobility of birds, detection at point counts was less likely to be as dependent upon the proportion of native plants, and many point count stations had a comparatively even mix of native and Acacia plants. Despite general findings that bird diversity is greater in more native habitat, Narango et al. (2017) found that regeneration of nonnative plants can increase food availability, especially compared to deforested habitat, keeping biomass relatively similar to that found in native habitat. Thus, while our data clearly document the higher quality of Sadhana Forest's intentional reforestation with native species, the increase in the water table that caused the Acacia to germinate and grow has also resulted in new forest cover.

Figure 7 Interpolated bird hotspot map of Sadhana Forest (see online version for colours)

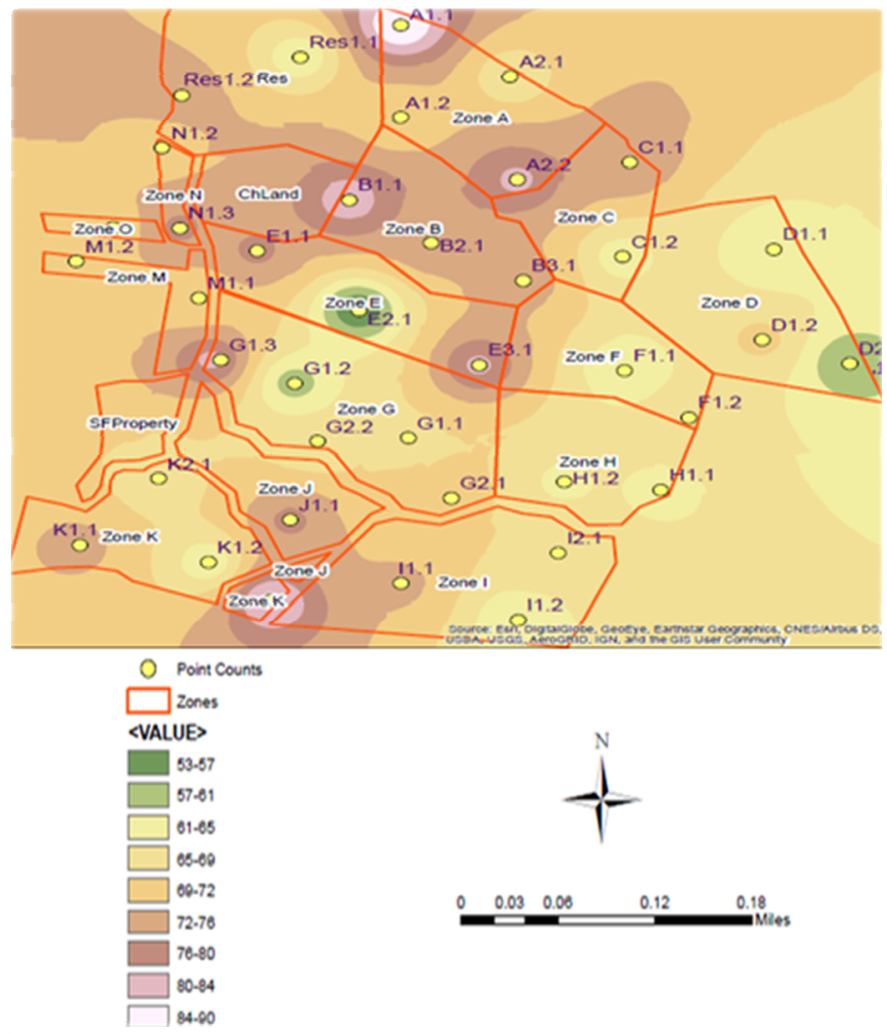

Notes: Green to light pink values show the estimated diversity of bird species at each point count census site compared to the amount of birds detected at neighbouring sites. Green represents the lowest estimation, orange is an intermediate colour, and light pink is representative of the highest amount of possible bird detects at a given location. The highest bird diversity mapped in this manner coincides with areas with greater proportions of native plants. 
This area was only inventoried during the dry season, but to document the full effects of this reforestation there is a need to conduct similar measures in the wet season to comprehensively document the reforestation impact. Narango et al. (2017) emphasise that bioinventories need to document how systems shift with time and seasonality. The reforestation efforts of Sadhana Forest have clearly created habitat that had existed in some previous form and that has attracted native fauna. More work can elucidate the benefits of regeneration through mere water retention compared to intentional plantings of native plant species (Prach and Hobbs, 2008). Our work documented presence-absence and does not measure the quality of different reforested areas. It is possible and perhaps even likely that birds are less likely to nest in areas dominated by Acacia given the lower amounts of arthropod prey. On the other hand, given the relatively small size of Sadhana Forest's holdings and the high mobility of birds, species may nest in Acacia-dominated areas if there is sufficient cover and adequate nesting substrate. To resolve this issue, more inventory work on all fauna sampled in this study is needed during the peak of the bird breeding season. Ideally, productivity measures could compare birds nesting in areas of different native-Acacia composition to examine more subtle ecological differences along this compositional gradient. Fichenscher et al. (2014) found greater numbers of pest and generalist invertebrate species in areas with more exotic shrub species in Northeastern US. Based upon our limited findings, there is likely to be positive faunal diversity outcomes by replacing Acacia with native species, but as suggested, this needs further investigation and the effort to eradicate Acacia must be considered.

Sadhana Forest's permaculture practices are restoring the historic ecological community. Similar efforts are occurring elsewhere in India (Balooni, 2003; Chen et al., 2019) and around the globe (Griscom and Ashton, 2011; Grossnickle and Ivetić, 2017), sometimes with mixed results (e.g., Cao et al., 2010; North et al., 2019). This bioinventory is important in documenting the outcomes of restoration given the major historic reduction in TDEF. McGeoch and Chown (1998) promote a multi-taxon approach to monitoring restoration success using multiple sampling methods like we have. Sadhana has essentially created a 28 ha TDEF woodland islet (Benayas et al., 2008) that could act as a seed source to the surrounding area depending upon future land use. Ongoing monitoring of such reforestation projects, particularly in dry systems, can be critical components to a multi-pronged approach to ecosystem restoration (Holl and Aide, 2011; Bowie et al., 2019). Thus, Sadhana Forest serves as a model for effective reforestation and ecosystem restoration.

\section{References}

Acheampong, E.O., Macgregor, C.J., Sloan, S. and Sayer, J. (2019) 'Deforestation is driven by agricultural expansion in Ghana's forest reserves', Scientific African, Vol. 5, p.e00146, DOI: https://doi.org/10.1016/j.sciaf.2019.e00146.

Babu, K.N., Biswajit, H. and Parthasarathy, N. (2019) 'Changes in tree diversity and carbon stock over a decade in two Indian tropical dry evergreen forests', Geology, Ecology, and Landscapes, DOI: 10.1080/24749508.2019.1696157.

Balooni, K. (2003) 'Economics of wastelands afforestation in India, a review', New Forests, Vol 26, No. 2, pp.101-136.

Benayas, J.M.R., Bullock, J.M. and Newton, A.C. (2008) 'Creating woodland islets to reconcile ecological restoration, conservation, and agricultural land use', Frontiers in Ecology and the Environment, Vol. 6, No. 6, pp.329-336. 
Binsangou, S., Ifo, S.A., Ibocko, L., Louvouandou, L., Tchindjang, M. and Koubouana, F. (2018) 'Urban growth and deforestation by remote sensing in the humid tropical forest of Congo Basin: case of Impfondo in Republic of Congo', American Journal of Environment and Sustainable Development, Vol. 3, No. 3, pp.46-54.

Bowie, M.H., Stokvis, E., Barber, K., Marris, J. and Hodge, S. (2019) 'Identification of potential invertebrate bioindicators of restoration trajectory at a quarry site in Hunua, Auckland, New Zealand', New Zealand Journal of Ecology, Vol. 43, No. 1, pp.1-11.

Cao, S., Tian, T., Chen, L., Dong, X., Yu, X. and Wang, G. (2010) 'Damage caused to the environment by reforestation policies in arid and semi-arid areas of China', Ambio, Vol. 39, pp.279-283, https://doi.org/10.1007/s13280-010-0038-z.

Carr, D. (2009) 'Population and deforestation: why rural migration matters', Progress in Human Geography, Vol. 33, No. 3, pp.355-378.

Chen, C., Park, T., Wang, X., Piao, S., Xu, B., Chaturvedi, R.K., Fuchs, R., Brovkin, V., Ciais, P., Fensholt, R., Tommervik, H., Bala, G., Zhu, Z., Nemani, R.R. and Myneni, R.B. (2019) 'China and India lead in greening of the world through land-use management', Nature Sustainability, Vol. 2, pp.122-129, DOI: https://doi.org/10.1038/s41893-019-0220-7.

Cristescu, R.H., Frère, C. and Banks, P.B. (2012) 'A review of fauna in mine rehabilitation in Australia: current state and future directions', Biological Conservation, Vol. 149, No. 1, pp.60-72.

Everard, M. (2018) 'The characteristics, representativeness, function and conservation importance of tropical dry evergreen forest on India's Coromandel Coast', Journal of Threatened Taxa, Vol. 10, No. 6, pp.11760-11769.

Fichenscher, J.L., Litvaitis, J.A., Lee, T.D. and Johnson, P.C. (2014) 'Insect responses to invasive shrubs: implications to managing thicket habitats in the Northeastern United States', Forest Ecology and Management, Vol. 322, pp.127-135, DOI: https://doi.org/10.1016/j.foreco. 2014.03.003.

Geist, H.J. and Lambin, E.F. (2002) 'Proximate causes and underlying driving forces of tropical deforestation: tropical forests are disappearing as the result of many pressures, both local and regional, acting in various combinations in different geographical locations', BioScience, Vol. 52, No. 2, pp.143-150.

Griscom, H.P. and Ashton, M.S. (2011) 'Restoration of dry tropical forests in Central America: a review of pattern and process', Forest Ecology and Management, Vol. 261, No. 10, pp.1564-1579.

Grossnickle, S.C. and Ivetić, V. (2017) 'Direct seeding in reforestation: a field performance review', Reforestation, Vol. 4, pp.94-142 [online] https://journal.reforestationchallenges.org/ index.php/REFOR/article/view/76.

Grove, R. (2002) 'Climatic fears: colonialism and the history of environmentalism', Harvard International Review, Vol. 23, No. 4, p.50.

Hobbs, R.J., Higgs, E., Hall, C.M., Bridgewater, P., Chapin III, F.S., Ellis, E.C. and Yung, L. (2014) 'Managing the whole landscape: historical, hybrid, and novel ecosystems', Frontiers in Ecology and the Environment, Vol. 12, No. 10, pp.557-564, DOI: 10.1890/130300.

Holl, K.D. and Aide, T.M. (2011) 'When and where to actively restore ecosystems?', Forest Ecology and Management, Vol. 261, No. 10, pp.1558-1563.

Holmes, R.T. and Schultz, J.C. (1988) 'Food availability for forest birds: effects of prey distribution and abundance on bird foraging', Canadian Journal of Zoology, Vol. 66, No. 3, pp.720-728.

Johnson, M.D. (2007) 'Measuring habitat quality: a review', The Condor, Vol. 109, No. 3, pp.489-504.

Kueffer, C. and Kaiser-Bunberry, C.N.K. (2014) 'Reconciling conflicting perspectives for biodiversity conservation in the Antropocene', Frontiers in Ecology and the Environment, Vol. 12, No. 2, pp.131-147. 
Mascaro, J., Hughes, R.F. and Schnitzer, S.A. (2012) 'Novel forests maintain ecosystem processes after the decline of the native tree species', Ecological Monographs, Vol. 82, No. 2, pp.221-228.

McGeoch, M.A. and Chown, S.L. (1998) 'Scaling up the value of bioindicators', Trends in Ecology and Evolution, Vol. 13, No. 2, pp.46-47.

Murcia, C., Aronson, J., Kattan, G.H., Moreno-Mateos, D., Dixon, K. and Simberloff, D. (2014) 'A critique of the 'novel ecosystem' concept', Trends in Ecology and Evolution, Vol. 29, No. 10, pp.548-553.

Muthumperumal, C. and Parthasarathy, N. (2016) 'Angiosperms, tropical dry evergreen forests of southern Coromandel Coast, India', Check List, Vol. 6, p.368, DOI: https://doi.org/10.15560/6.3.368.

Narango, D.L., Tallamy, D.W. and Marra, P.P. (2017) 'Native plants improve breeding and foraging habitat for an insectivorous bird', Biological Conservation, Vol. 213, pp.42-50, DOI: https://doi.org/10.1016/j.biocon.2017.06.029.

Nithaniyal, S., Vassou, S.L., Poovitha, S., Raju, B. and Parani, M. (2017) 'Identification of species adulteration in traded medicinal plant raw drugs using DNA barcoding', Genome, Vol. 60, No. 2, pp.139-146.

North, M.P., Stevens, J.T., Greene, D.F., Coppoletta, M., Knapp, E.E., Latimer, A.M., Restaino, C.M., Tompkins, R.E., Welch, K.R., York, R.A., Young, D.J.N., Axelson, J.N., Buckley, T.N., Estes, B.L., Hager, R.N., Long, J.W., Meyer, M.D., Ostoja, S.M., Safford, H.D., Shive, K.L., Tubbesing, C.L., Vice, H., Walsh, D., Werner, C.M. and Wyrsch, P. (2019) 'Tamm review: reforestation for resilience in dry western U.S. forests', Forest Ecology and Management, Vol. 432, pp.209-224, DOI: https://doi.org/10.1016/ j.foreco.2018.09.007.

Oyetunji, P.O., Ibitoye, O.S., Akinyemi, G.O., Fadele, O.A. and Oyediji, O.T. (2020) 'The effects of population growth on deforestation in Nigeria: 1991-2016', Journal of Applied Sciences and Environmental Management, Vol. 24, No. 8, pp.1329-1334.

Palmer, M.A., Bernhardt, E.S., Schlesinger, W.H., Eshleman, K.N., Foufoula-Georgiou, E., Hendryx, M.S., Lemly, A.D., Likens, G.E., Loucks, O.L., Power, M.E., White, P.S. and Wilcock, P.R. (2010) 'Mountaintop mining consequences', Science, Vol. 327, No. 5962, pp.148-149.

Prach, K. and Hobbs, R.J. (2008) 'Spontaneous succession versus technical reclamation in the restoration of disturbed sites', Restoration Ecology, Vol. 16, No. 3, pp.363-366.

Prach, K., Řehounková, K., Řehounek, J. and Konvalinková, P. (2011) 'Ecological restoration of Central European mining sites: a summary of a multi-site analysis', Landscape Research, Vol. 36, No. 2, pp.263-268.

Pyles, M.V., Prado-Junior, J.A., Magnago, L.F., de Paula, A. and Meira-Neto, J.A. (2018) 'Loss of biodiversity and shifts in aboveground biomass drivers in tropical rainforests with different disturbance histories', Biodiversity and Conservation, Vol. 27, No. 12, pp.3215-3231.

R Core Team (2018) R: A Language and Environment for Statistical Computing, R Foundation for Statistical Computing, Vienna, Austria [online] https://www.R-project.org/ (accessed September 2018).

Reddy, C.S., Jha, C.S., Dadhwal, V.K., Krishna, P.H., Pasha, S.V., Satish, K.V., Dutta, K., Saranya, K.R.L., Rakesh, F., Rajashekar, G. and Diwakar, P.G. (2016) 'Quantification and monitoring of deforestation in India over eight decades (1930-2013)', Biodiversity and Conservation, Vol. 25, No. 1, pp.93-116.

Reddy, C.S., Saranya, K.R.L., Pasha, S.V., Satish, K.V., Jha, C.S., Diwakar, P.G. and Murthy, Y.K. (2018) 'Assessment and monitoring of deforestation and forest fragmentation in South Asia since the 1930s', Global and Planetary Change, Vol. 161, pp.132-148, DOI: https://doi.org/10.1016/j.gloplacha.2017.10.007.

Tallamy, D.W. (2004) 'Do alien plants reduce insect biomass?', Conservation Biology, Vol. 18, No. 6, pp.1689-1692. 